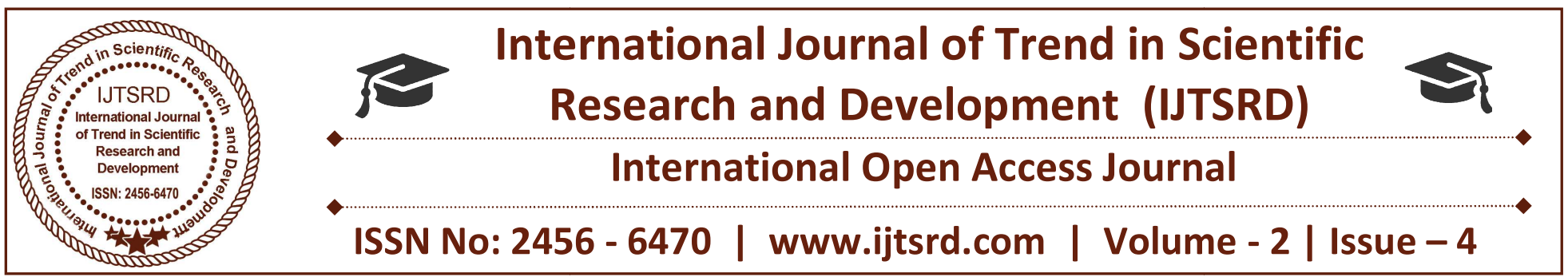

\title{
Peer To Peer File Transfer with Torrent
}

\author{
Mr. Samir Ashok Mohite \\ Student, Bharti Vidyapeeth Institute of Management and Information Technology \\ CBD Belapur, Navi Mumbai, Maharashtra, India
}

\begin{abstract}
Peer to peer data transfer received a lot of attention in recent years. A peer-to-peer network allows users to communicate or send data over a network without any server. A peer is any computer client or user. So that it called Peer-to-peer (P2P) networking as there is no server, all data transfer process is going between those peers only. Peer-to-peer file sharing means distribution of files over a network without participation of any server, in which the files are located on individual's machine and distributed with other members of the network.
\end{abstract}

These type of networking softwares like Bit Torrent founded as the piracy method of choice in the early years butthen Supreme Court announced decision[2] to the closure of many torrent sites as they encourages illegally sharing of copyrighted content like games, music, setup files and mostly movies.

\section{INTRODUCTION}

An example of a p2pfile transfer that an efficient and time saving alternative to the server-client file transfer solution is torrenting. It is a distributed system, which has the ability to change the base of file distribution process. One can directly download data from other's computer with the help of torrent files. The centralized tracker is present over there to provide the different address list over available peers for appropriate file transfer. A torrent client is program that uses and applies the Torrent protocol. Each client can send as well asreceive any type of computer file over a network at the same time, using the protocol. In this protocol there is no actual source for the files, people download those files which are uploaded by others peers.

\section{Participants:-}

Seeds: Peers or users who have already downloaded the files, and are uploading them for any other peer. Seeding is where you let your Torrent client open even after you've finished your download and helping to distribute that file to other peers which is requesting for that file.[1]

Leeches: is any peer that does not have the entire file and is downloading the file without uploading. Leeches always haspoor share ratio, and itspecifies that there is downloading much more than their uploading, so they automatically decrease downloading speed of other peers. Leeches do not leave their Torrent client open to seed the file after they download the whole file. [1]

Trackers: A tracker is like centralized server that keeps track of which seeds and peers are in the system or you can say "swarm". Swarm is set of all peers sharing a file. The tracker only manages connections of one peer to another, it does not have any knowledge of the contents of the files being uploaded. 


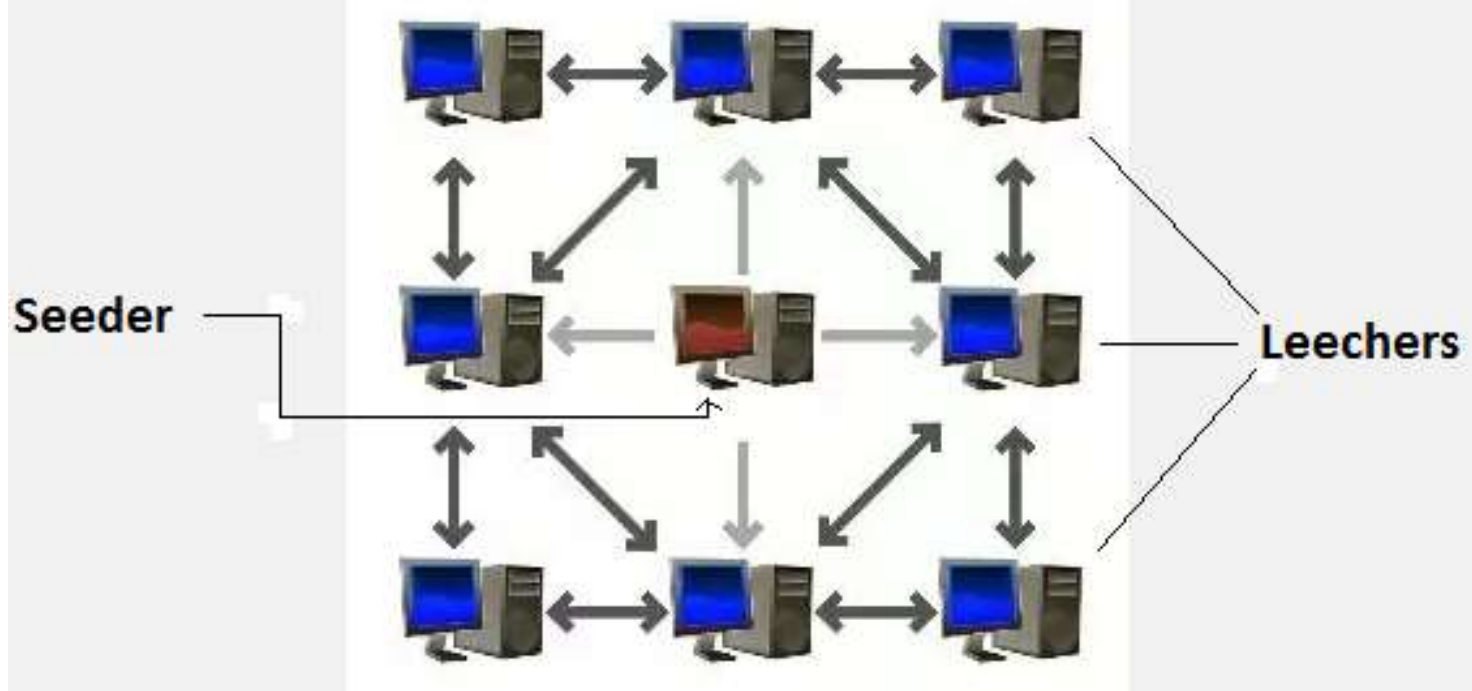

So as we can see in above figure, in the middle there is seeder present and he's uploading file for other users. After completion of download one peer, he becomes seeder and start uploading that file for newly entered user in network.

\section{Working Overview:-}

To distribute the content of file or group of files, a peer first creates a file called a "torrent" e.g. Interstaller. torrent, with extension (.torrent). This file contains metadata about the actual file to be distributed and about the tracker, the computer that manages the file distribution.

Peers or client that wants to download the file must first obtain that specific torrent file for it. Because of that torrent file has metadata about trackers so it is easy to connect to the specified tracker, which tells them from which other peers to download the file. There are pieces of every file, it refers to the torrented files being divided up into equal specific sized pieces e.g. $512 \mathrm{kB}, 1 \mathrm{MB}, 2 \mathrm{MB}$ or $4 \mathrm{MB}$. It get easy to share in divided file format and automatically saves more time. Though both transfer content of file over a network, so this type of downloading is differs from a classical client-server download as with HTTP requests

Bit Torrent makes many small data requests (like in pieces) over different IP connections to different machines which leads to achieve efficiency, in other words it saves more efforts and time to download such files

\section{Safe or unsafe?}

Every user who use torrent application has their own mindset due to which the answer for the question could be different. There arechances of cybercrime or attack means hacking through torrenting is possible, because this torrent client share your IP address with other users who are downloading the same file or content over a network. Now if your IP address has been tracked by any other person or a hacker then they can hack your whole system and you may lose your important content.

You can safely use any torrent client by hiding online identity means by hiding your IP address. This could be done with the help of VPN services. VPN stand for Virtual Private Network which place important role here to hide your original online identity and provides you a fake identity of your choice (fake IP address). This has been done by changing the IP address of your system and internet temporary. By using VPN, no one can tracks you including the hackers because they see a wrong or fake IP address that you has changed.

\section{Torrent sites are banned:}

There is nothing inherently illegal about Torrent, rather it has proven that it is a good option for file transferring by maintaining efficiency. Torrenting is legal or illegal is directly depends on what you are downloading. Downloading copyrighted ('C)')material that you don't have a license is definitely consider as crime. 
Indian IT Act 2000 announced by Government and References:-

directly mentions that anything from downloading

1. https://www.quora.com/

copyrighted content is illegal in India. So, technically, you can be jailed for using such Torrent sites in India,

2. http://www.cyberlawsindia.net/ if you're not careful about your downloading content.

[2]

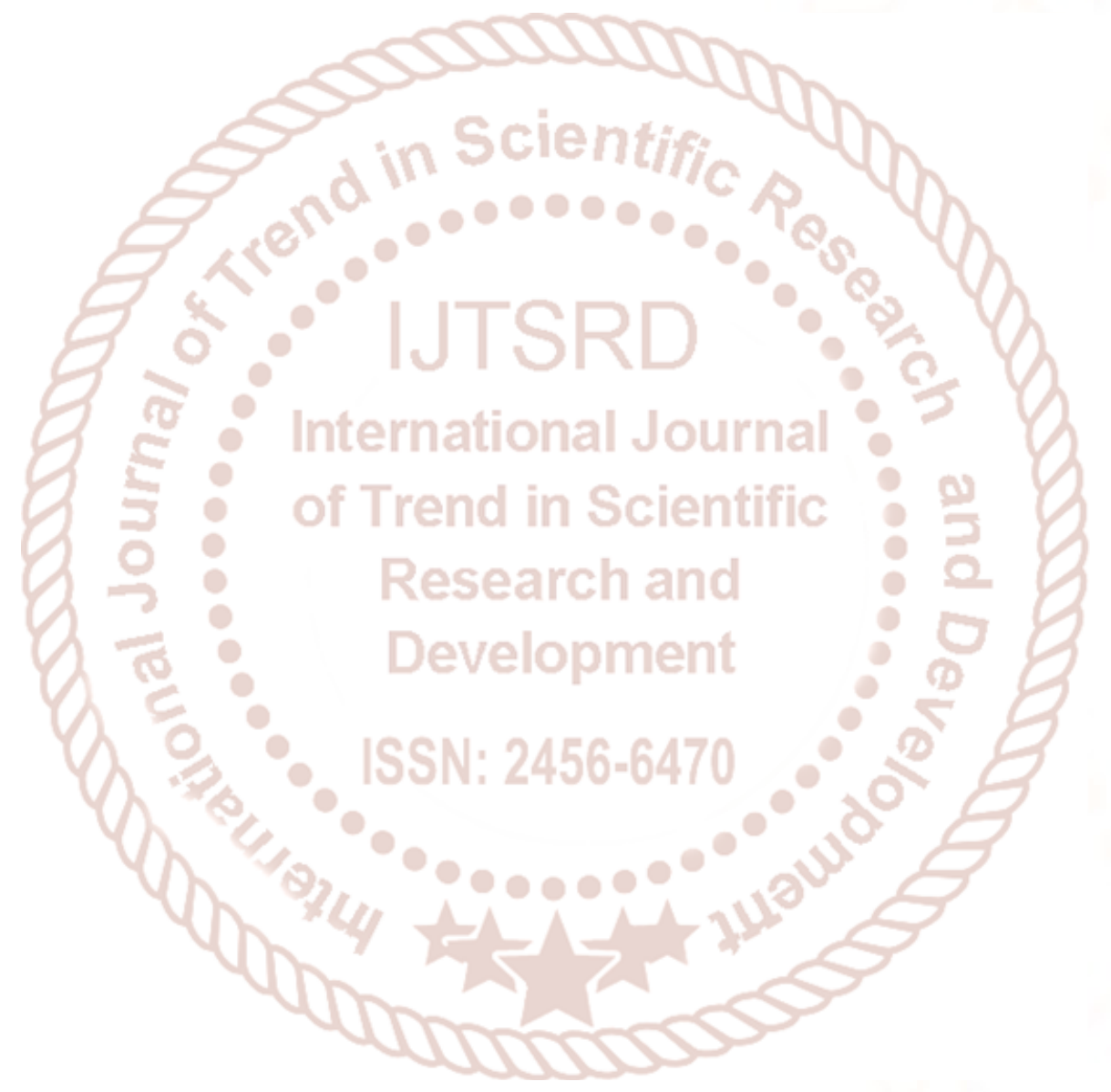

